\title{
Improvement of Accuracy in Flow Immunosensor System by Introduction of Poly-2-[3-(methacryloylamino)propylammonio] ethyl 3-aminopropyl Phosphate
}

\author{
Yusuke Fuchiwaki, ${ }^{1,2}$ Mikito Yasuzawa, ${ }^{1}$ Norimichi Futagami, ${ }^{1}$ and Kotaro Rikitake \\ ${ }^{1}$ Department of Chemical Science and Technology, Graduate School of Advanced Technology and Science, The University of Tokushima, \\ 2-1 Minamijosanjima, Tokushima-shi, Tokushima 770-8506, Japan \\ ${ }^{2}$ Health Research Institute, National Institute of Advanced Industrial Science and Technology (AIST), 2217-14 Hayashicho, Takamatsu, \\ Kagawa 761-0395, Japan
}

Correspondence should be addressed to Yusuke Fuchiwaki, yu-fuchiwaki@aist.go.jp

Received 9 November 2010; Revised 23 March 2011; Accepted 15 June 2011

Academic Editor: Jiri Homola

Copyright (c) 2011 Yusuke Fuchiwaki et al. This is an open access article distributed under the Creative Commons Attribution License, which permits unrestricted use, distribution, and reproduction in any medium, provided the original work is properly cited.

In order to improve the accuracy of immunosensor systems, poly-2-[3-(methacryloylamino)propylammonio] ethyl 3-aminopropyl phosphate (poly-3MAm3AP), which includes both phosphorylcholine and amino groups, was synthesized and applied to the preparation of antibody-immobilized beads. Acting as an antibody-immobilizing material, poly-3MAm3AP is expected to significantly lower nonspecific adsorption due to the presence of the phosphorylcholine group and recognize large numbers of analytes due to the increase in antibody-immobilizing sites. The elimination of nonspecific adsorption was compared between the formation of a blocking layer on antibody-immobilized beads and the introduction of a material to combine antibody with beads. Determination with specific and nonspecific antibodies was then investigated for the estimation of signal-to-noise ratio. Signal intensities with superior signal-to-noise ratios were obtained when poly-3MAm3AP was introduced. This may be due to the increase in antibody-immobilizing sites and the extended space for antigen-antibody interaction resulting from the electrostatic repulsion of poly-3MAm3AP. Thus, the application of poly-3MAm3AP coatings to immunoassay beads was able to improve the accuracy of flow immunosensor systems.

\section{Introduction}

Immunosensors quantify analytes based on binding reactions between antibody and antigen, and various immunosensing methods have been reported for the quantitative determination of a broad range of analytes of clinical, medical, biotechnological, and environmental significance [1-4]. However, nonspecific adsorption can lead to a decline in sensor accuracy and sensitivity, and this has limited the further development of immunosensors toward practical application. Nonspecific adsorption decreases the signal-tonoise $(\mathrm{S} / \mathrm{N})$ ratio, preventing the improvement of immunosensor systems. Therefore, eliminating nonspecific adsorption is crucial in realizing a practical immunosensor system.
Blocking reagents such as nonspecific sera, detergents, milk proteins, bovine serum albumin (BSA), and ovalbumin are typically used to reduce nonspecific adsorption [5-9]. On the other hand, blocking reagents also block the binding sites for specific interaction between antibodies and antigens [10]; thus, blocking methods interfere with sensitive and accurate determination, despite their efficacy in reducing nonspecific adsorption. Moreover, the composition of nonspecific adsorption reducing layers with blocking reagents typically requires additional technical know-how for incubation in sensing. Such incubation processes prevent application to rapid and continuous measurements. Therefore, alternative methods for reducing nonspecific adsorption have been investigated. 
In recent years, hydrophilic polymers such as poly-2methacryloyloxyethyl phosphorylcholine (poly-MPC) have been utilized as materials for reducing protein sorption [1114]. It has been reported that the adsorption of protein onto a film of phosphorylcholine derivatives is limited due to the presence of free water at the polymer-liquid interface [13, 14]. However, the introduction of phosphorylcholine-containing polymer to the antigen-antibody interface did not increase the signal intensity caused by the specific reaction, while nonspecific adsorption was eliminated. Therefore, analyte selectivity is improved by its introduction, but the sensitivity and accuracy are unsatisfactory. To overcome this issue, the construction of an interface that not only reduces nonspecific adsorption but also increases the specific reaction-based determination is of intrinsic importance.

Poly-2-[3-(methacryloylamino)propylammonio] ethyl 3aminopropyl phosphate (poly-3MAm3AP), which has a phosphorylcholine moiety for nonspecific adsorption removal and an amino group for covalent bonding with antibody, was synthesized and applied to antigen-antibody interfaces. As the phosphorylcholine group has both hydrophilic and electrolytic properties, poly-3MAm3AP would be effective in preventing entanglement with other molecules. Therefore, the introduction of antibody attached to poly3MAm3AP would be effective for trapping larger numbers of antigens due to the introduction of large numbers of antibodies.

The reaction area (reactor) for antigen (analyte) recognition and the detection area (detector) for signal translation were separated with the construction of an immunosensor system. Otherwise, recognizing an antigen on the surface of the detector as in our previous reports would cause an increase in background noise due to adsorption of high molecular weight protein $[4,10]$. By separating the reactor and detector, antigen recognition and signal translation should not affect one another in the detection process.

The effects of nonspecific adsorption removal were evaluated as follows: (i) formation of a nonspecific adsorption preventing layer on antibody-immobilized substrate by incubation with blocking reagents; (ii) immobilization of antibodies on a nonspecific adsorption-preventing polymer formed on the substrate.

Poly-MPC and poly-3MAm3AP were used as blocking reagents and antibody-immobilizing material, respectively. BSA, which is known to be useful as both a blocking reagent and an antibody-immobilizing material, was used for comparison. In addition, an acrylamide polymer contain ing positively charged choline and amino groups, poly4-[3-(acryloylamino)propylammonio]-1-butylamine (poly3AAm1BA), which differs from poly-3MAm3AP in that poly3AAm1BA does not contain a phosphoric ester, was also employed as the antibody-immobilizing material for comparison of the polymer electrolyte effect.

Luteinizing hormone (LH), which is useful for sterility treatment, was employed as a model antigen and was labeled with fluorescein (F-LH) for sensitive fluorescence detection $[15,16]$. Two monoclonal IgG class antibodies, antiluteinizing hormone antibody (anti-LH) and antihemoglobin anti body (anti-hemo), were employed for the estimation of nonspecific adsorption levels, as the difference obtained between F-LH and anti-hemo will be only nonspecific adsorption. Antibodies were directly immobilized onto agarose gel beads or by the mediation of antibody-immobilizing material and packed into a reactor column. The other conditions for fabricating the flow system were as described previously $[4,17,18]$.

Compared with recent technologies that allow the accuracy and sensitivity to be increased, this paper describes an approach for enhancing immunosensor accuracy and sensitivity by introducing antibodies immobilized on beads coated with poly-3MAm3AP [19-23]. Furthermore, poly3MAm3AP beads do not require incubation with blocking reagents or bound/free separation.

We truly believe that this system will give a significant impact on immunosensor technologies in future.

\section{Experimental}

2.1. Reagents and Materials. Luteinizing hormone (LH), monoclonal IgG class anti-LH, and antihemoglobin antibodies were kindly provided by ROHTO Pharmaceutical Co. Bovine serum albumin (BSA, 96\%-99\%) was obtained from Sigma Chemical. Commercial 2-chloro1,3,2-dioxaphospholane was purified by vacuum distillation. Commercial 2,2' -azobisisobutyronitrile (AIBN) was recrystallized from methanol. All other solvents were purchased as the best commercial grade and dried over molecular sieves (4 $\AA)$ prior to use. All other chemicals and solvents were of commercial grade and used without further purification. Standard stock solutions containing antigens were prepared with phosphate buffer solution (PBS, $\mathrm{pH}$ 7.0) and were stored at $4{ }^{\circ} \mathrm{C}$. Antibodies were stored in a frozen state, and standard solutions were prepared daily with PBS solution as needed. All aqueous solutions were prepared in deionized distilled water. Affi-Gel $\mathrm{Hz}$ hydrazide gel beads were purchased from Bio-Rad Laboratories and were used to immobilize antibodies. LH was modified with fluorescein isothiocyanate to prepare fluorescein-labeled LH (F-LH) for use as an antigen in the flow immunosensor system.

2.2. Synthesis of Poly-3MAm3AP. Hydrophilic phosphorylcholine-containing polymer, poly-2-[3-(methacryloylamino) propylammonio] ethyl 3-aminopropyl phosphate (poly-3MAm3AP), was synthesized as shown in Scheme 1. To a stirred dry THF solution of $\mathrm{N}$-(3-hydroxypropyl) phthalimide and triethylamine, 2-chloro-2-oxo-1,3,2-dioxophosphorane was added at $-20^{\circ} \mathrm{C}$ and the reaction mixture was maintained at $0^{\circ} \mathrm{C}$ for $2 \mathrm{~h}$. After filtration of triethylamine hydrochloride, the filtrate and THF solutions were evaporated in vacuo in a stream of nitrogen. The resulting product was reacted with $\mathrm{N}, \mathrm{N}$-(dimethylamino propyl)methacrylamide in acetonitrile at $80^{\circ} \mathrm{C}$ for $20 \mathrm{~h}$, yielding 2-[3-(methacryloylamino)propylammonio] ethyl 3phthalimidopropyl phosphate (3MAm3PP). 3MAm3PP was 


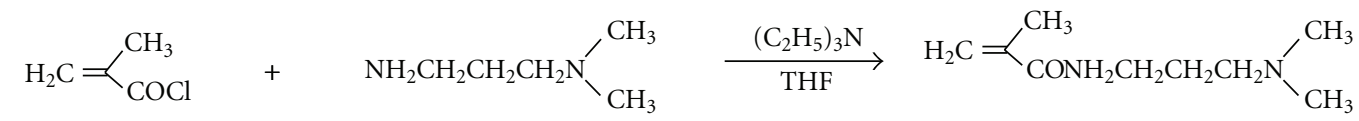

Methacrylic chloride $\quad N, N$-dimethyl-1,3-propanediamine

$N$-(dimethylaminopropyl)methacrylamide (PMAm)<smiles>CN1C(=O)c2ccccc2C1=O</smiles>

$N$-(3-hydroxypropyl)-phthalimide<smiles>O=P1(Cl)OCCO1</smiles>

2-chloro-2-oxo-1,3,2dioxaphospholane

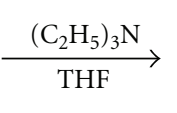<smiles>CCOP1(=O)OCCO1</smiles><smiles>C=C(C)C(=O)NCCC[NH+](C)CCOP(=O)(OCC)OCCN1C(=O)c2ccccc2C1=O</smiles><smiles>CCCCC(C)(C)C(=O)NCCC[NH+](C)CC</smiles>

Poly-3MAm3PP<smiles>CCN1C(=O)c2ccccc2C1=O</smiles>

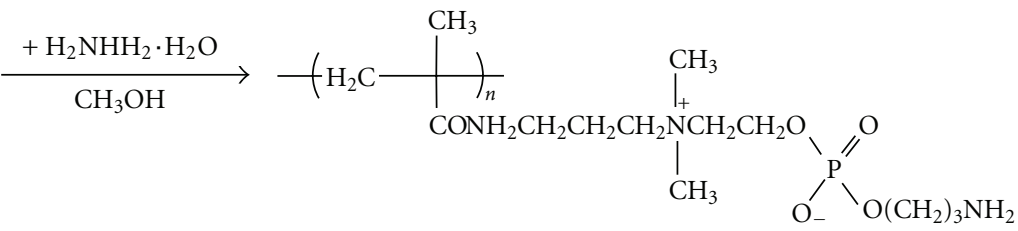

Poly-3MAm3AP

Scheme 1: Reaction route for synthesis of poly-3MAm3AP.

then polymerized at $80^{\circ} \mathrm{C}$ for $20 \mathrm{~h}$ in acetonitrile using AIBN as an initiator, and poly-3MAm3PP was obtained. Finally, poly-3MAm3PP was subjected to a hydrolysis reaction with hydrazine hydrate in methanol, and the phthalimide protecting groups were removed to afford poly-3MAm3AP.

2.3. Synthesis of Poly-3AAm1BA. Synthesis of acrylamide polymer containing both positive amino groups and positively charged choline groups and lacking phosphate groups (poly-3AAm1BA) was performed according to Scheme 2. $N$-(Dimethylaminopropyl) acrylamide and $N$-(3-bromoxypropyl) phthalimide were reacted in benzene and were then polymerized in acetonitrile. The phthalimide protecting groups were removed to afford the acrylamide polymer of poly-4-[3-(acryloylamino) propylammonio]-1-butylamine, poly-3AAm1BA.

2.4. Preparation of Antibody-Immobilized Beads. Antibodyimmobilized beads for blocking reagent treatment were prepared according to instruction manual provided by Bio-Rad Laboratories [24], and the beads were packed into the reactor column $(50 \times 3.0 \mathrm{~mm}$ I.D. EYELA $)$. Antibody-immobilized beads attached through polymers were prepared according to the procedure in Scheme 3. Carboxylic groups were introduced to the surface of the beads by succinic anhydride, and the activated ester was prepared using $N$-ethyl- $N$-(3- 


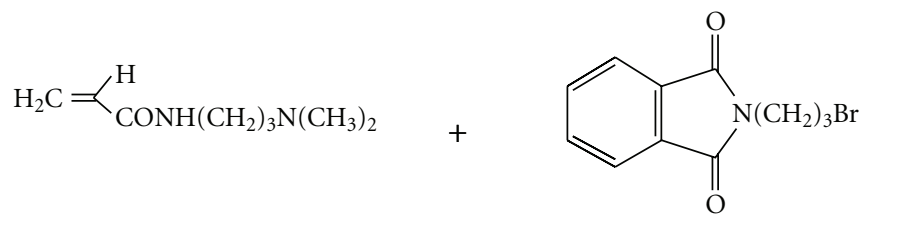<smiles>C=C(N)CCCN(C)CCN1C(=O)c2ccccc2C1=O</smiles>

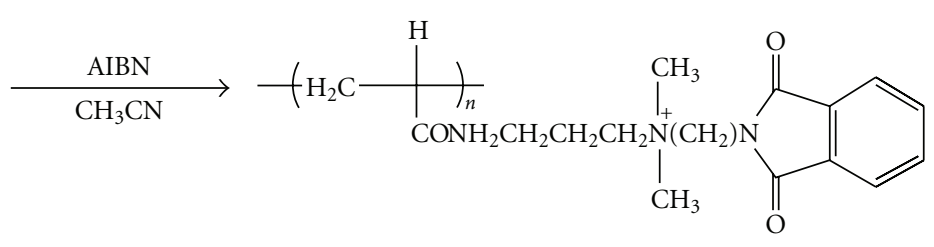

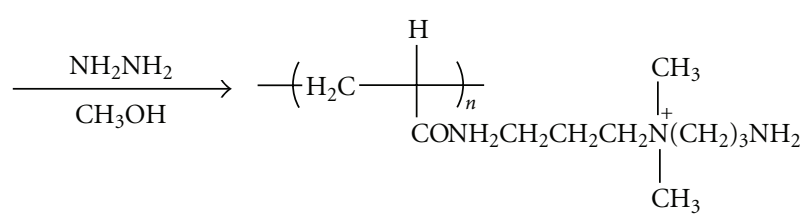

Poly-3AAm1BA

Scheme 2: Reaction route for synthesis of poly-3AAm1BA.

dimethylaminopropyl) carbodiimide (EDC) and $N$-hydroxy succinimide (NHS). Beads were reacted with the polymers to form amide bonds. Prior to IgG antibody binding to the polymer, the antibody was partially purified for maximizing the binding capacity and oxidized the carbohydrate moieties on the Fc region to form aldehydes [24]. Finally, the antibodies were attached to the polymers, and the prepared antibody-immobilized beads were attached through polymers.

2.5. Principle and Apparatus of Reactor/Detector Separated Flow System. Measurements using the reactor/detector separated flow system were performed as follows. First, F-LH dissolved in PBS was injected onto the reactor column with peristaltic pumps (EYELA MP-3 microtube pump) and was trapped by the antibody-immobilized beads. Second, acetic acid solution was injected to cleave the antigen-antibody binding. Third, free F-LH antigen in acetic acid solution was mixed with $0.02 \mathrm{M}$ sodium hydrate solution through a flowing process before loading onto the optical detector, as the fluorescence of F-LH was only minimally detected in solutions of less than $\mathrm{pH}$ 6.0. Finally, the F-LH solution flowed into the detector and fluorescence intensity was measured. All of the measurements were automatically performed with the fabricated flow system at room temperature, as shown in Figure 1. BSA blocking procedure was performed as reported previously $[17,18,25]$. Tween 20 solution $(0.5 \%)$ was employed for cleaning both the quartz crystal cell used in the optical detector and the flow path in the system. Position valve $\mathrm{C}$ was set as the flow channel in order to avoid loading the Tween 20 solution onto the reactor column. Thus, the adsorption of F-LH onto the quartz cell could be washed between measurements by loading the Tween 20 solution from position valve $\mathrm{B}$.

\section{Results and Discussion}

3.1. Study of F-LH Detection in Reactor and Detector Separated Immunosensor System. A calibration curve was produced using various concentrations of F-LH with antiLH antibody-immobilized bead (anti-LH) column and antihemoglobin (anti-hemo) columns (Figures 2(a)-2(c)). Measurement was performed three times against each concentration. To reduce nonspecific adsorption, BSA and poly-MPC were used as blocking reagents. As a result, the reactor and detector separated immunosensor system was able to detect different concentrations of F-LH, and their fluorescence res- 


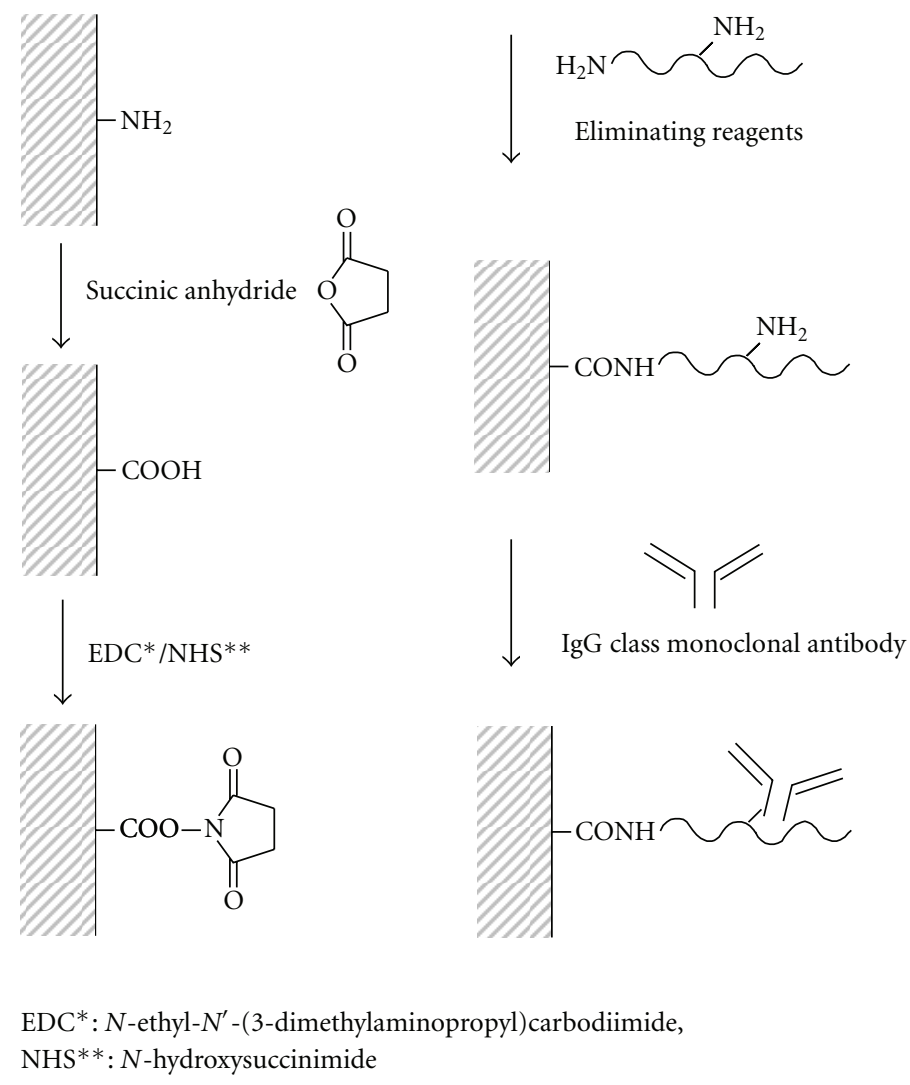

Scheme 3: Preparation procedure for antibody-immobilized beads including nonspecific adsorption-reducing reagents.

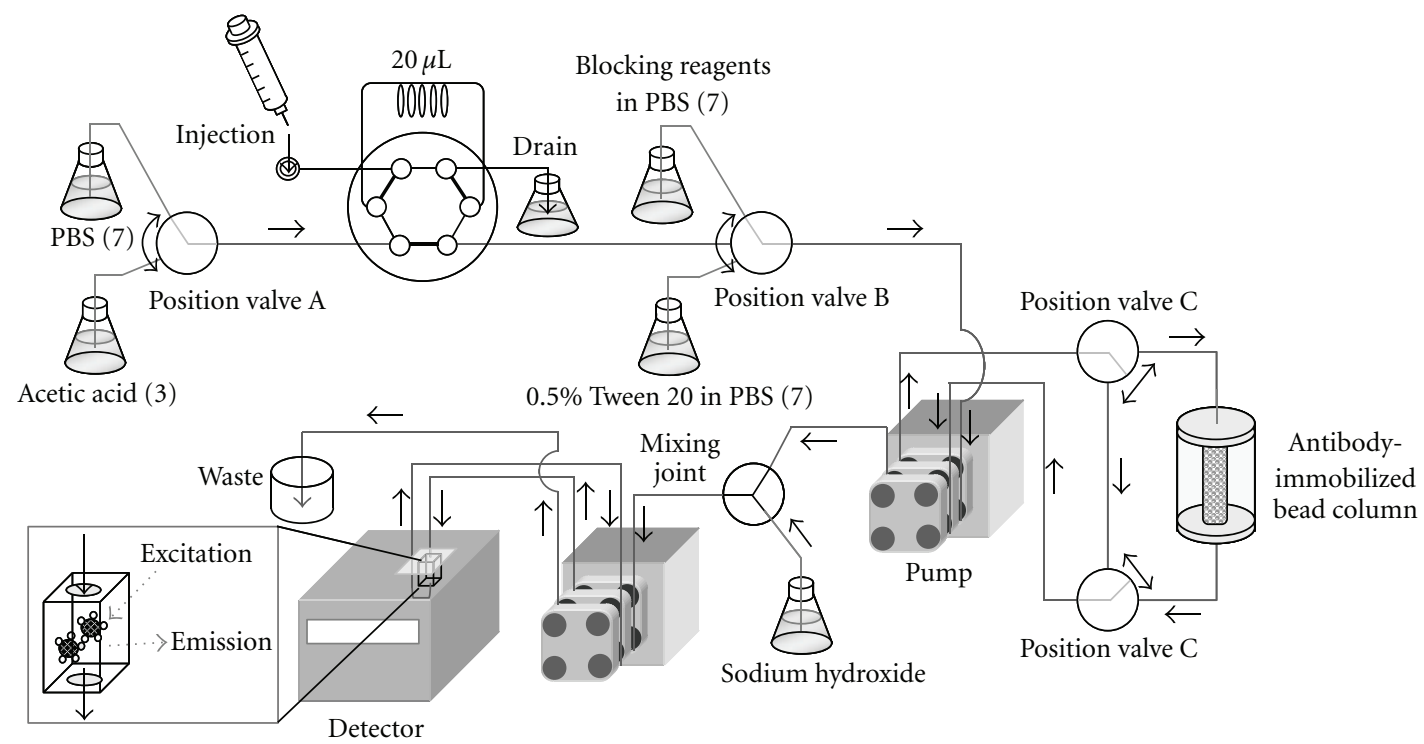

Figure 1: Flow immunosensor system.

ponses depended on the linear increase of F-LH concentration up to $3.5 \mu \mathrm{g} \mathrm{cm}^{-3}$ (Figure 2). Although the antihemo columns contained no specific binding sites for F$\mathrm{LH}$, they all exhibited linear increases with increasing $\mathrm{F}-$ LH concentration, even when blocking reagents were used. Moreover, the poly-MPC blocking layer had no effect on eliminating nonspecific adsorption, while BSA had some effect observed. This indicates that nonspecific adsorption occurred on the anti-hemo-immobilized beads.

3.2. Effects of Poly-3MAm3AP Beads on Nonspecific Adsorption. We planned to immobilize antibodies covalently on 


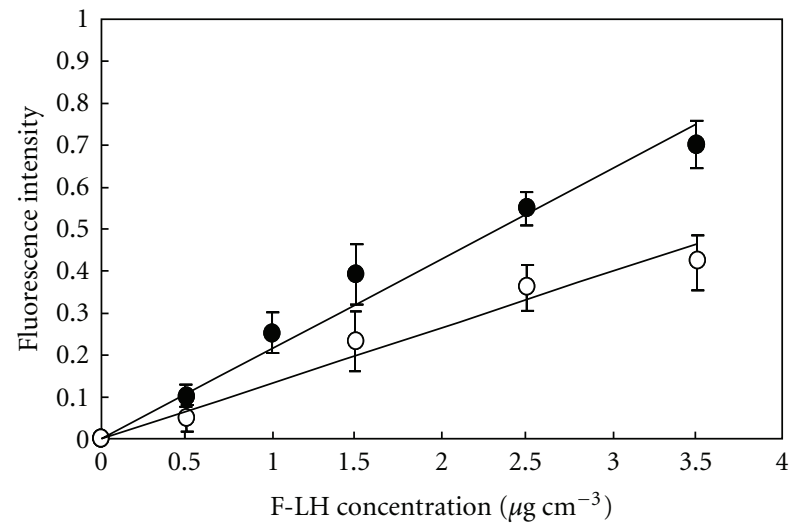

(a)

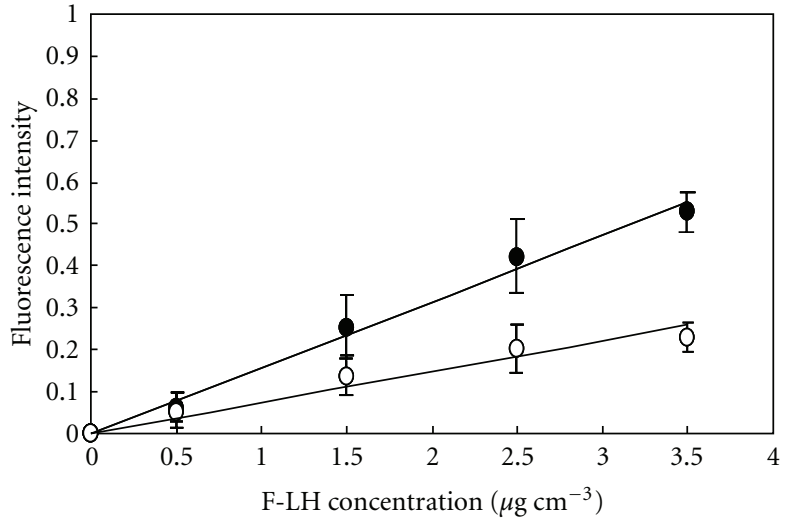

(b)

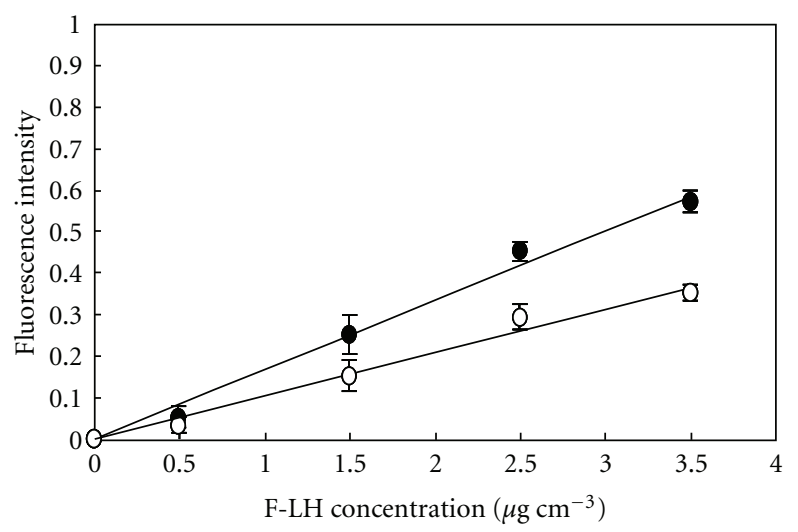

(c)

FIGURE 2: Relationship between different concentrations of F-LH and fluorescence intensity, under (a) nonblocking conditions, (b) with BSA, and (c) with poly-MPC. Measurements were performed with anti-LH-(closed symbols) and anti-hemoglobin-(open symbols) immobilized beads, respectively.

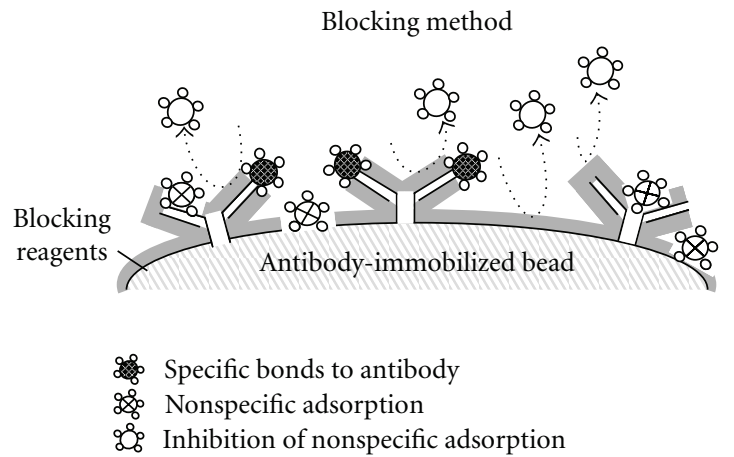

(a)

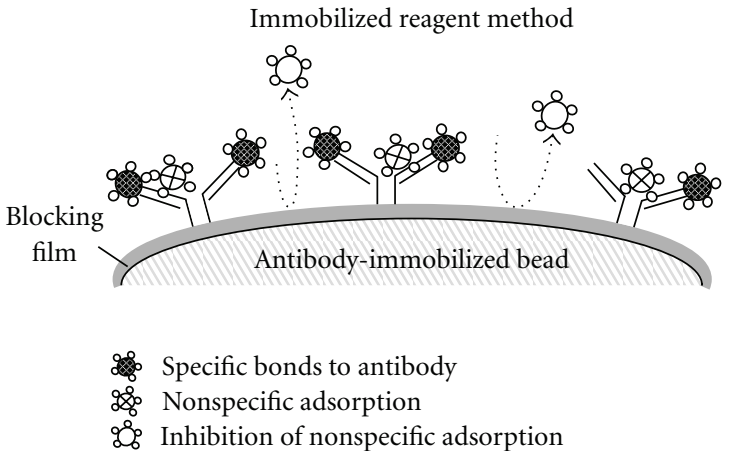

(b)

FIgURE 3: Removal of nonspecific adsorption with (a) blocking method and (b) immobilized reagent method.

the beads via poly-3MAm3AP, which reduces nonspecific adsorption (Figure 3). Such a method would not require complex procedures or technical knowledge, blocking of anti body-antigen interaction sites, or release of film for nonspecific adsorption removal.

Poly-3MAm3AP, which had amino groups for covalent attachment with antibodies and beads, was synthesized and used as a phosphorylcholine-containing polymer. Acrylamide polymer (poly-3AAm1BA), which contains both amino and positively charged choline groups, but no negatively charged phosphoric ester, and the known blocking reagent BSA were employed for comparison. Calibration curves were fabricated as shown in Figure 4, and the data were obtained three times for each concentration. The slopes of 


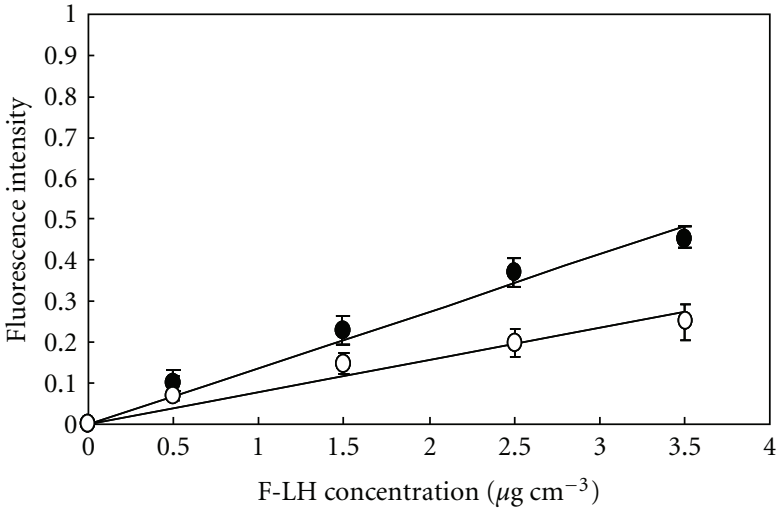

(a)

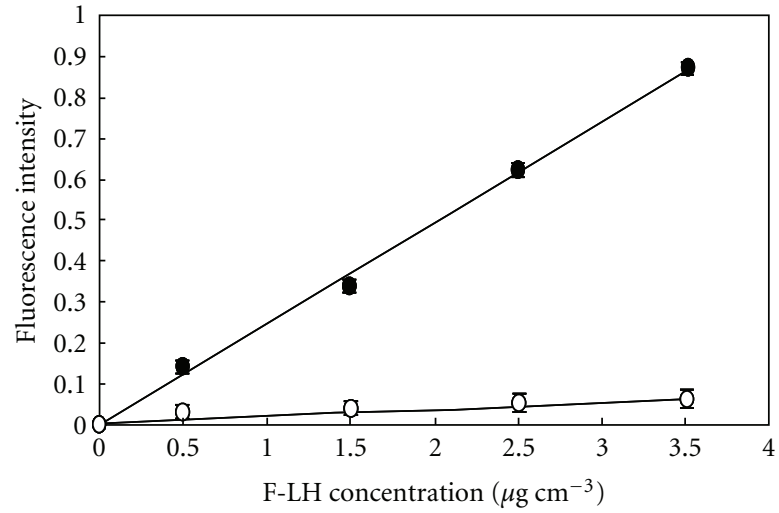

(b)

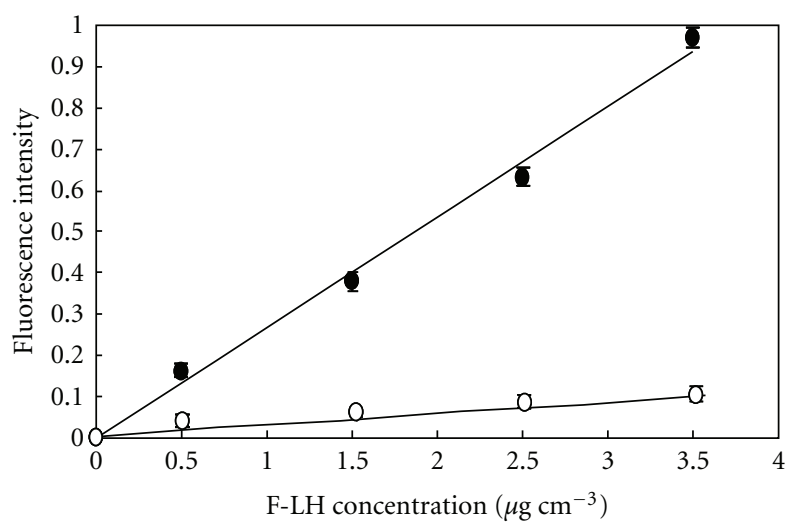

(c)

FIGURE 4: Relationship between concentration of F-LH and fluorescence intensity. (a) BSA membrane, (b) poly-3MAm3AP, and (c) poly3AAm1BA were, respectively, immobilized on the surface of antibody-immobilized beads before the antibody-binding process.

calibration curves obtained from anti-hemo-immobilized beads were significantly lower when poly-3MAm3AP and poly-3AAm1BA were employed (Figure 4). This indicates that nonspecific adsorption was successfully reduced (Figures $4(\mathrm{~b})$ and $4(\mathrm{c})$ ). The effectiveness of poly-3MAm3AP was attributed to the phosphorylcholine derivative which removed nonspecific adsorption due to the presence of free water. The most likely explanation for the successful reduction of nonspecific adsorption by poly-3AAm1BA is that positively charged poly-3AAm1BA produced a highly hydrophilic surface $[10,16]$. However, the strongly positively charged poly-3AAm1BA may limit the reduction of nonspecific adsorption when compared with nearly neutral poly3MAm3AP, as negatively charged antigens will adsorb poly3AAm1BA by electrostatic attraction. The isoelectric point of the model sample LH was in the range of 6.51-6.77 [26], and the antigen-antibody reaction was performed in $\mathrm{pH} 7.0$ solution; thus, the measurement conditions were suitable for poly-3AAm1BA to act as an effective antibody-immobilized material. On the other hand, the BSA-immobilized beads scarcely reduced the nonspecific adsorption.

For the slopes of anti-LH, notable results were obtained. The slopes of poly-3MAm3AP and poly-3AAm1BA were higher than those of the BSA-immobilized beads (Figure 4).
This is attributable to the fact that the number of the antibodies immobilized to the beads increased by modifying those polymers around the beads (Figure 5(a)). Although the antibodies were directly immobilized on the surface of the beads, a substantial number of antibodies could be introduced to the small area on the beads by using these polymers, as shown in Figure 5(a). Generally, the densely immobilized polymer chains would become entangled, making it difficult for the host molecule attached to the polymer to bind the guest molecule. On the other hand, phosphatidylcholine analogues are reported to have an overall positive charge due to the stronger positive charge of the choline groups, producing hydrophilic properties [2729]. As poly-3AAm 1BA possessed only the positively charged choline groups, the strong repulsion between the polymer chains and the antigen F-LH readily allowed access to the antibodies on the polymers. The anti-LH slope of poly$3 \mathrm{MAm} 3 \mathrm{AP}$ was therefore similar to that of poly-3AAm1BA. This indicates that poly-3MAm3AP was able to capture a larger quantity of antigen F-LH due to the repulsion between polymers preventing entanglement (Figure 5(b)). In addition, acrylamide polymers not containing choline groups did not show such increases in anti-LH slope. Therefore, sufficient space for the antigen-antibody reaction 


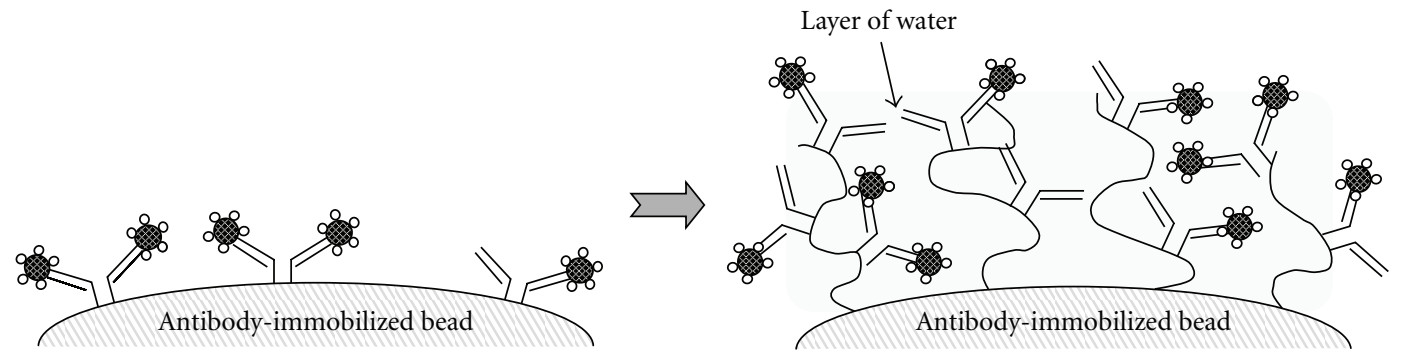

(a)
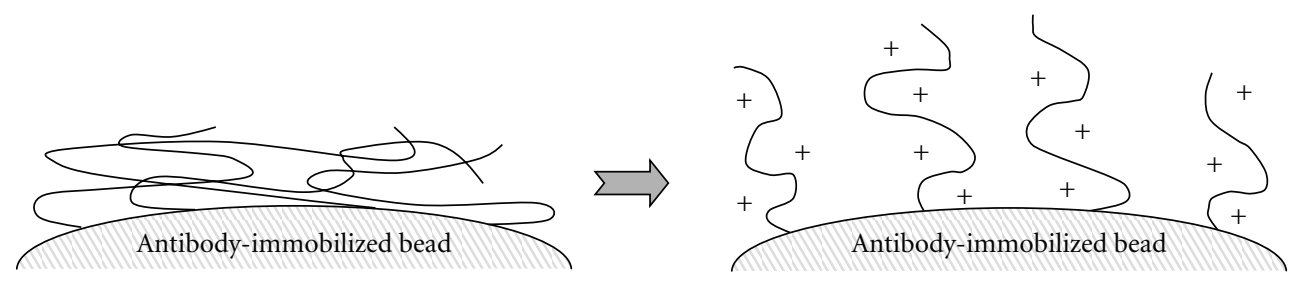

(b)

FIGURE 5: Image on the surface of poly-3MAm3AP-immobilized beads. (a) Increase in immobilized antibodies by introducing poly3MAm3AP to eliminate nonspecific adsorption. (b) Swelling effect of electrolytic properties of poly-3MAm3AP.

TABLE 1: Comparison of the slopes and variations obtained from calibration curves of the sensor system.

\begin{tabular}{lcccccc}
\hline Method & \multicolumn{3}{c}{ Blocking } & & \multicolumn{2}{c}{ Immobilization } \\
& None & BSA & Poly-MPC & BSA & Poly-3MAm3AP & Poly-3AAm1BA \\
\hline Anti-LH & 0.214 & 0.158 & 0.168 & 0.138 & 0.246 & \pm .298 \\
Variation* & \pm 0.112 & \pm 0.121 & \pm 0.087 & \pm 0.080 & 0.032 & 0.014 \\
Anti-hemo & 0.133 & 0.074 & 0.104 & 0.078 & 0.057 & 0.021 \\
Ratio** & 0.622 & 0.468 & 0.619 & 0.565 & 0.070 \\
\hline
\end{tabular}

${ }^{*}$ Average relative deviation obtained from calibration plot of anti-LH.

**Anti-hemo/Anti-LH.

may remain on the surface of poly-3MAm3AP-immobilized beads, and F-LH antigens could easily access the anti-LH antibodies attached to the polymer for specific binding.

3.3. Evaluation of Accuracy of Immunosensor System. In order to evaluate sensor accuracy, the variations and slopes obtained from the calibration curves were analyzed and compared, as shown in Table 1. Variation was calculated from 12 measurements. The effects of specific bonds were estimated based on the ratio of the slope of the anti-hemo curve to that of the anti-LH curve (Table 1). Among the blocking methods, BSA was effective in reducing nonspecific adsorption, as shown by a lower ratio than the nonblocking case; however, the variation became wider and the slope of anti-LH decreased. The ratios of poly-3AAm1BA and poly-3MAm3AP were nearer to zero when compared with the others due to almost all nonspecific adsorption removal. Moreover, the variations with poly-3AAm1BA and poly-3MAm3AP were also smaller than the others due to their allowing specific interactions between antibodies and antigens, while reducing nonspecific adsorption. This indicates that immunosensors using these hydrophilic polymerimmobilized beads would be able to more accurately detect antigen F-LH. Generally, nonspecific adsorption causes both a decrease in the $\mathrm{S} / \mathrm{N}$ ratio and a decrease in sensor accuracy. However, blocking methods also decrease these factors, in addition to requiring complex technical knowledge to fully function, thus making them unsuitable for use in an accurate flow-sensor with automatic determination and various sensing applications.

The results obtained for poly-3MAm3AP beads confirmed satisfying effects for both the reduction of nonspecific adsorption and the increase in signal intensity caused by specific reactions.

\section{Conclusion}

Poly-3MAm3AP was introduced to the antigen-antibody interface in an effort to improve the accuracy of the immunosensor system. Anti-LH and anti-hemo were employed for the estimation of nonspecific adsorption levels. Poly3MAm3AP-immobilized beads exhibited a high anti-LH slope and a low anti-hemo slope, resulting in a considerably lower ratio of anti-LH to anti-hemo. This indicates that poly3MAm3AP markedly reduces nonspecific adsorption and specifically trapped large amounts of F-LH. The introduction 
of poly-3MAm3AP to the interface significantly reduced nonspecific adsorption with high accuracy in the fabricated immunosensor system. This immunosensor device may assist in the development for other biosensing devices by substituting other biological receptors for antibodies.

\section{Acknowledgments}

The authors are grateful to ROHTO Pharmaceutical Co., Ltd., Japan, for providing Luteinizing hormone (LH), monoclonal IgG class anti-LH and antihemoglobin antibodies. This work was supported by a Grant-in-Aid for Scientific Research (22710125).

\section{References}

[1] M. Y. Liu, A. M. Xydakis, R. C. Hoogeveen et al., "Multiplexed analysis of biomarkers related to obesity and the metabolic syndrome in human plasma, using the Luminex-100 system," Clinical Chemistry, vol. 51, no. 7, pp. 1102-1109, 2005.

[2] X. Ao, R. F. Rotundo, D. J. Loegering, and J. A. Stenken, "In vivo microdialysis sampling of cytokines produced in mice given bacterial lipopolysaccharide," Journal of Microbiological Methods, vol. 62, no. 3, pp. 327-336, 2005.

[3] T. Krakauer, "A sensitive, specific immunobioassay for quantitation of human interleukin 6," Journal of Immunoassay, vol. 14, no. 4, pp. 267-277, 1993.

[4] M. Yasuzawa, H. Hamada, K. Oga, and Y. Fuchiwaki, "Design and construction of automated amperomeric immunosensing system," International Journal of Modern Physics B, vol. 17, no. 8-9, pp. 1211-1216, 2003.

[5] B. Batteiger, W. J. V. Newhall, and R. B. Jones, "The use of Tween 20 as a blocking agent in the immunological detection of proteins transferred to nitrocellulose membranes," Journal of Immunological Methods, vol. 55, no. 3, pp. 297-307, 1982.

[6] S. M. Spinola and J. G. Cannon, "Different blocking agents cause variation in the immunologic detection of proteins transferred to nitrocellulose membranes," Journal of Immunological Methods, vol. 81, no. 1, pp. 161-165, 1985.

[7] J. W. Haycock, "Polyvinylpyrrolidone as a blocking agent in immunochemical studies," Analytical Biochemistry, vol. 208, no. 2, pp. 397-399, 1993.

[8] V. V. Shmanai, "Blocking of non-specific sorption in ELISA on formylated polystyrene beads," Journal of Immunoassay, vol. 20, no. 1-2, pp. 13-30, 1999.

[9] S. Sakaki, Y. Iwasaki, N. Nakabayashi, and K. Ishihara, "Watersoluble 2-methacryloyloxyethyl phosphorylcholine copolymer as a novel synthetic blocking reagent in immunoassay system," Polymer Journal, vol. 32, no. 8, pp. 637-641, 2000.

[10] P. Irwin, A. Gehring, S. I. Tu, and C. Y. Chen, "Blocking nonspecific adsorption of native food-borne microorganisms by immunomagnetic beads with $\iota$-carrageenan," Carbohydrate Research, vol. 339, no. 3, pp. 613-621, 2004.

[11] T. McPherson, A. Kidane, I. Szleifer, and K. Park, "Prevention of protein adsorption by tethered poly(ethylene oxide) layers: experiments and single-chain mean-field analysis," Langmuir, vol. 14, no. 1, pp. 176-186, 1998.

[12] C. R. Jenney and J. M. Anderson, "Effects of surface-coupled polyethylene oxide on human macrophage adhesion and foreign body giant cell formation in vitro," Journal of Biomedical Materials Research, vol. 44, no. 2, pp. 206-216, 1999.
[13] K. Ishihara, R. Aragaki, T. Ueda, A. Watenabe, and N. Nakabayashi, "Reduced thrombogenicity of polymers having phospholipid polar groups," Journal of Biomedical Materials Research, vol. 24, no. 8, pp. 1069-1077, 1990.

[14] H. Kitano, A. Kawasaki, H. Kawasaki, and S. Morokoshi, "Resistance of zwitterionic telomers accumulated on metal surfaces against nonspecific adsorption of proteins," Journal of Colloid and Interface Science, vol. 282, no. 2, pp. 340-348, 2005.

[15] C. A. Marquette and L. J. Blum, "State of the art and recent advances in immunoanalytical systems," Biosensors and Bioelectronics, vol. 21, no. 8, pp. 1424-1433, 2006.

[16] P. J. Conroy, S. Hearty, P. Leonard, and R. J. O'Kennedy, "Antibody production, design and use for biosensor-based applications," Seminars in Cell and Developmental Biology, vol. 20, no. 1, pp. 10-26, 2009.

[17] Y. Fuchiwaki, H. Hamada, and M. Yasuzawa, "Stability evaluation of antibody-immobilized column for immunosensor using optical procedure," Chemical Sensors, vol. 18, supplement B, pp. 64-66, 2002.

[18] Y. Fuchiwaki, K. Rikitake, N. Futagami, and M. Yasuzawa, "Reproducibility evaluation of immunosensor with antibodyimmobilized beads column (II)," Chemical Sensors, vol. 19, supplement B, pp. 64-66, 2003.

[19] A. Ramanavicius, N. Ryskevic, Y. Oztekin et al., "Immunosensor based on fluorescence quenching matrix of the conducting polymer polypyrrole," Analytical and Bioanalytical Chemistry, vol. 398, no. 7-8, pp. 3105-3113, 2010.

[20] A. Kausaite-Minkstimiene, A. Ramanaviciene, J. Kirlyte, and A. Ramanavicius, "Comparative study of random and oriented antibody immobilization techniques on the binding capacity of immunosensor," Analytical Chemistry, vol. 82, no. 15, pp. 6401-6408, 2010.

[21] Z. Zhong, M. Li, D. Xiang et al., "Signal amplification of electrochemical immunosensor for the detection of human serum IgG using double-codified nanosilica particles as labels," Biosensors and Bioelectronics, vol. 24, no. 7, pp. 2246-2249, 2009.

[22] T. Zhang, R. Yuan, Y. Chai, K. Liu, and S. Ling, "Study on an immunosensor based on gold nanoparticles and a nanocalcium carbonate/Prussian blue modified glassy carbon electrode," Microchimica Acta, vol. 165, no. 1-2, pp. 53-58, 2009.

[23] H. Vaisocherová, W. Yang, Z. Zhang et al., "Ultralow fouling and functionalizable surface chemistry based on a zwitterionic polymer enabling sensitive and specific protein detection in undiluted blood plasma," Analytical Chemistry, vol. 80, no. 20, pp. 7894-7901, 2008.

[24] "Affi-Gel Hz Immunoaffinity Kit Instruction Manual Catalog Number 153-6060 Bio-Rad Laboratories," http://wolfson.huji. ac.il/purification/PDF/affinity/BIORAD_Immunoaffinity_kit. pdf.

[25] H. Ogi, Y. Fukunishi, H. Nagai, K. Okamoto, M. Hirao, and M. Nishiyama, "Nonspecific-adsorption behavior of polyethylenglycol and bovine serum albumin studied by $55-\mathrm{MHz}$ wireless-electrodeless quartz crystal microbalance," Biosensors and Bioelectronics, vol. 24, no. 10, pp. 3148-3152, 2009.

[26] http://www.abcam.co.jp/Luteinizing-Hormone-alpha-antibody-ME-116-ab 9506.html.

[27] M. Yasuzawa, T. Nakaya, and M. Imoto, "Polyacrylamides containing phosphatidylcholine," Macromolecular Science-Chemistry, vol. 23, no. 8, pp. 963-972, 1986.

[28] M. Yasuzawa, T. Nakaya, and M. Imoto, "Synthesis and properties of vinyl polymers containing both vitamin $\mathrm{E}$ and 
phos zphatidylcholine analogous moieties," Makromolekulare Chemie, Rapid Communications, vol. 6, pp. 727-731, 1985.

[29] M. Yasuzawa, T. Nakaya, and M. Imoto, "Synthesis and properties of vinyl polymers containing both cholesterol and phosphatidylcholine analogous moieties," Makromolekulare Chemie, Rapid Communications, vol. 6, pp. 721-726, 1985. 

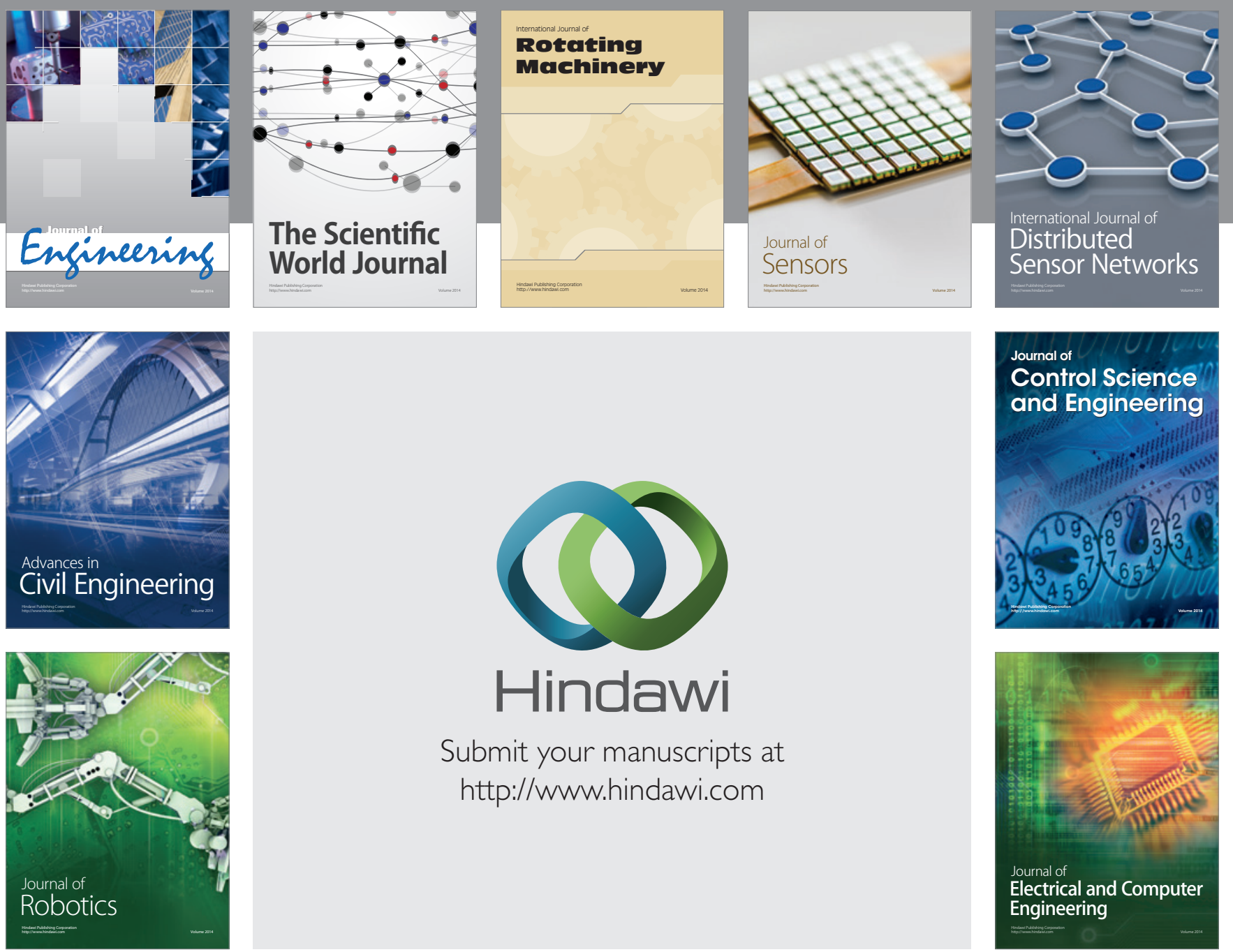

Submit your manuscripts at

http://www.hindawi.com
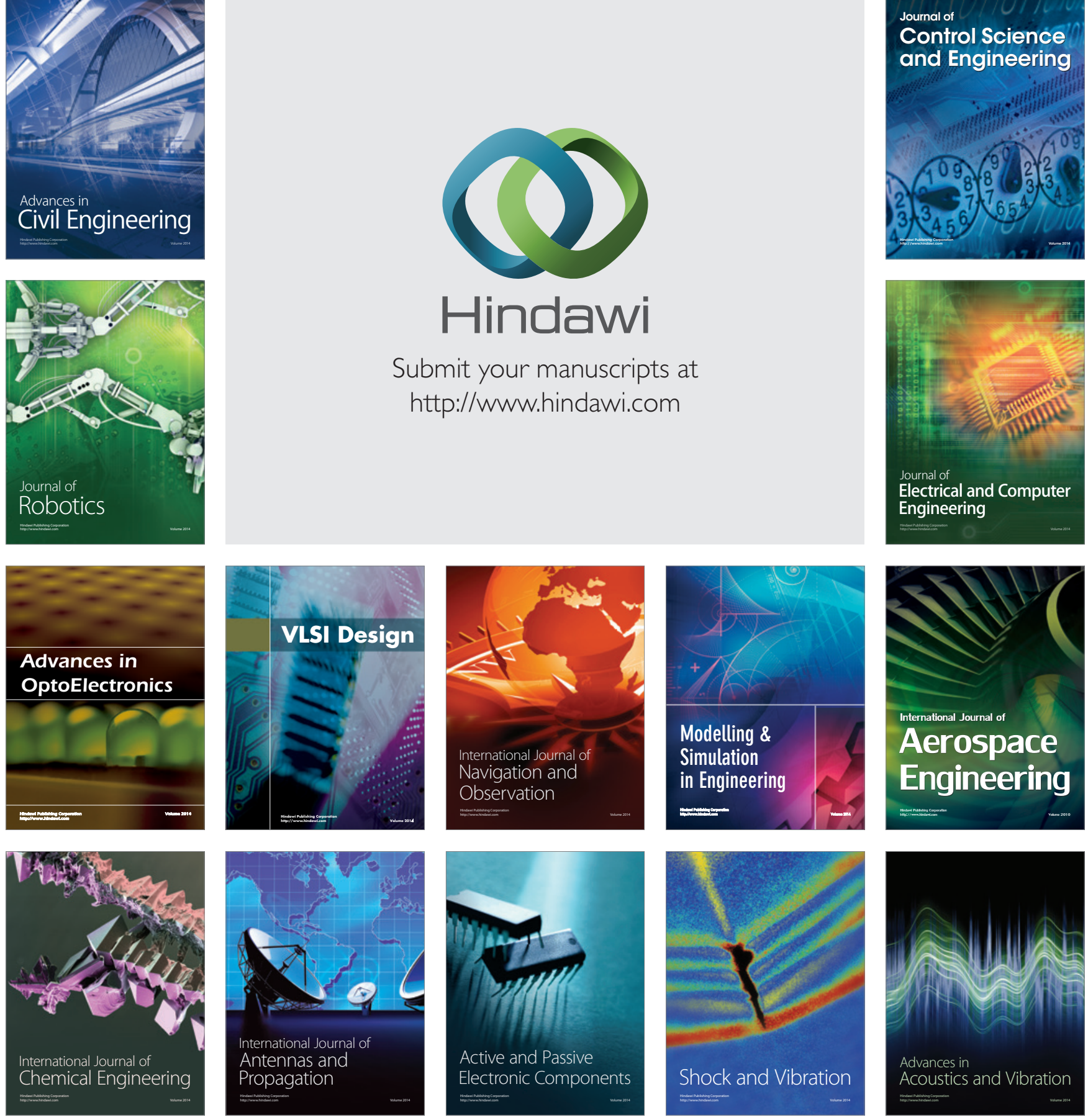